\title{
Comparison of echocardiography and radiology in the diagnosis of aortic root dilatation in Marfan's syndrome and in syphilis
}

\author{
NICHOLAS G KOUNIS AND K CONSTANTINIDIS \\ From the Cardiac Department, Royal Victoria Hospital, Belfast and MRC Pneumoconiosis Unit, \\ Llandough Hospital, Penarth, Glamorgan
}

ABSTRACT Aortic root dilatation was sought by echocardiography and radiology in 500 consecutive patients referred to an echocar diography laboratory from cardiac or medical clinics and wards. Forty-four of these patients $(8.8 \%)$ had false echocardiographic evidence of aortic root dissection, but no clinical evidence of the disease. These false findings were attributed to the changing of the transducer angulation, post-stenotic dilatation, calcific aortic stenosis, generalised artetiosclerosis, and thin reverberations behind the posterior wall echo. In one patient with Marfan's syndrome, the chest radiograph was normal and a diagnosis was established by echocardiography. In another patient with syphilis, the dilatation was seen in the chest radiograph while echocardiography revealed a normal proximal aorta. In syphilis the dilatation begins distally and because of the intervening lung tissue echocardiography is unhelpful. Echocardiograms easily detect the aortic valve ring while radiographs show the more distal ascending aorta. These observations may be of added value in the diagnosis of aortic root disease.

Localised or diffuse dilatation of the aorta may be caused by syphilis, arteriosclerosis, trauma, congenital defects (osteogenesis imperfecta, Ehlers-Danlos syndrome), mycotic infections, ankylosing spondylitis, aortic stenosis, and cystic medionecrosis associated with Marfan's syndrome, coarctation of the aorta, pregnancy, myxoedema, and lathyrism. ${ }^{1}$ The aortic root may also be affected by several of the above causes and this can be revealed by conventional chest radiographs. Echocardiography has been recommended as a non-invasive method for the diagnosis and measurement of the extent of the dilatation. ${ }^{2}$ When the aortic root dilatation is situated within the cardiac shadow plain radiographs may fail to reveal it. ${ }^{3}$ In this case invasive methods, such as contrast cine-angiography, are necessary for the evaluation of this condition. ${ }^{45}$ It has not been determined whether echocardiographic studies can detect aortic root dilatation which is not apparent on plain radiographs. ${ }^{6}$ Syphilitic aortic root dilatation begins distally in the ascending aorta and may spare the region of the sinuses of Valsalva. ${ }^{3}$ The dilatation which

Address for reprint requests: Dr NG Kounis, 48 Maizonos Street, Queen Olga's Square, Patras, Greece. complicates Marfan's syndrome maximally involves, and may be limited to, the area containing the annulus of the aortic valve and sinuses of Valsalva. ${ }^{3-5} 7$

The purpose of this report is to determine the usefulness of echocardiography and conventional radiography in the diagnosis of aortic root dilatation complicating syphilis and Marfan's syndrome.

\section{Methods}

The echocardiographic findings of the aortic root and the available radiographic and angiographic data of 500 patients were reviewed. The patients were referred from outpatient clinics, a coronary care unit, and medical or cardiac wards. Echocardiograms were performed at the bedside or in the echocardiography laboratory. A commercially available echocardiograph (Smith-Kline-Ekoline) with $2.25 \mathrm{MHz}$ transducer focused at $5 \mathrm{~cm}$ was used. A simultaneous electrocardiogram was recorded in a strip chart recorder at a speed of $50 \mathrm{~mm} / \mathrm{s}$. The patients were placed in a supine position or turned on the left side at an angle of $30^{\circ}$ to the bed and with two pillows behind the head. The transducer was placed on the third, 
fourth, or fifth left intercostal space parasternally in a medial and cephalic direction pointing to the right shoulder until the two parallel echoes from the anterior and posterior aortic walls were seen. Echocardiographic measurements of the aortic root dimension were made at a level just above the aortic valve leaflets at the end of systole. The normal aortic root dimension, determined by echocardiography and measured between the inner mangins of the anterior and posterior aortic walls is in our laboratory $20-37 \mathrm{~mm}$. Posteroanterior and left lateral chest radiographs were obtained. Angiographic studies of the aortic arch were performed with the Seldinger technique using a mixture of meglumine diatrizoate $66 \%$ and sodium diatrizoate $10 \%$ (Urographin $76 \%$ ).

\section{Results}

The final diagnoses in the 500 patients are shown in table 1 . Seventy-seven patients had echocardiographic evidence of aortic root dilatation (table 2 ). Of these patients, 29 had minimal echocardiographic aortic root dilatation (up to $45 \mathrm{~mm}$ ). None of these patients had suffered from any disease known to be associated with aortic dilatation. The chest radiographs were normal and catheterisation data were not available. Three patients had aortic root dissection detected echocardiographically immediately after their admission via a mobile coronary care unit. Radiographic widening of the mediastinum was apparent in these patients. The diagnosis of dissection was confirmed by angiography, during operation, or at necropsy (two patients). Fortyfour patients had false echocardiographic evidence $^{2}$ of aortic root dissection. There was no clinical evidence of the disease in these patients.

Table 1 Final diagnoses of the patients in study. Several patients had more than one underlying clinical condition

\begin{tabular}{ll}
\hline Diagnosis & Number of cases \\
\hline Myocardial infarction & 150 \\
Chronic ischaemic heart disease & 101 \\
Hypertension & 18 \\
Hyperthyroidism & 3 \\
Myxoedema & 5 \\
Hypertrophic obstructive cardiomyopathy & 3 \\
Mitral stenosis & 49 \\
Aortic valve stenosis & 46 \\
Combined mitral and aortic valve disease & 22 \\
Marfan's syndrome & 1 \\
Syphilis & 1 \\
Mitral valve prosthesis & 16 \\
Aortic valve prosthesis & 12 \\
Mitral and aortic valve prosthesis & 9 \\
Diabetes & 18 \\
Bronchial asthma & 8 \\
Normal (no apparent illness) & 38 \\
\hline
\end{tabular}

Table 2 Cause of echocardiographic aortic root dilatation in 77 patients

\begin{tabular}{ll}
\hline Cause & Number of patients \\
\hline Minimal aortic dilatation (no obvious cause) & 29 \\
Arteriosclerotic aortic dissection & 3 \\
False diagnosis of aortic dissection & 44 \\
Marfanoid aortic dilatation & 1 \\
\hline
\end{tabular}

We attributed this echocardiographic appearanceto the changing of the transducer angulation (16) patients), poststenotic dilatation caused by aortic valve disease (15 patients), calcific aortic stenosis $x$ (eight patients), generalised arteriosclerosis (three $w$ patients), and thin reverberations behind the $\sigma$ posterior wall echo (two patients). One patient $\vec{g}$ was found to have Marfan's syndrome and $a_{0}$ normal aorta in the conventional chest radio- graph, but a dilated aortic root on echocardio- $\vec{c}$ grams and aortograms. Another patient with a history of syphilis had dilatation of the ascending ${ }^{\mathscr{D}}$ aorta and aneurysms detected by aortography, $\overrightarrow{0}$ but a normal echocardiographic aortic root.

\section{PATIENT 1}

A 23-year-old woman was referred to the cardiology department complaining of palpitations. She had been previously diagnosed as $\frac{}{\varnothing}$ having Marfan's syndrome. Echocardiographic $\underset{\vec{F}}{\vec{*}}$ studies showed dilatation of the aortic root measuring $48 \mathrm{~mm}$ at the end of systole (fig 1) and holosystolic posterior motion of the mitral leaflets compatible with mitral valve prolapse. Posteroanterior and lateral chest radiographs (fig 2)。 showed marked thoracic scoliosis with a normal

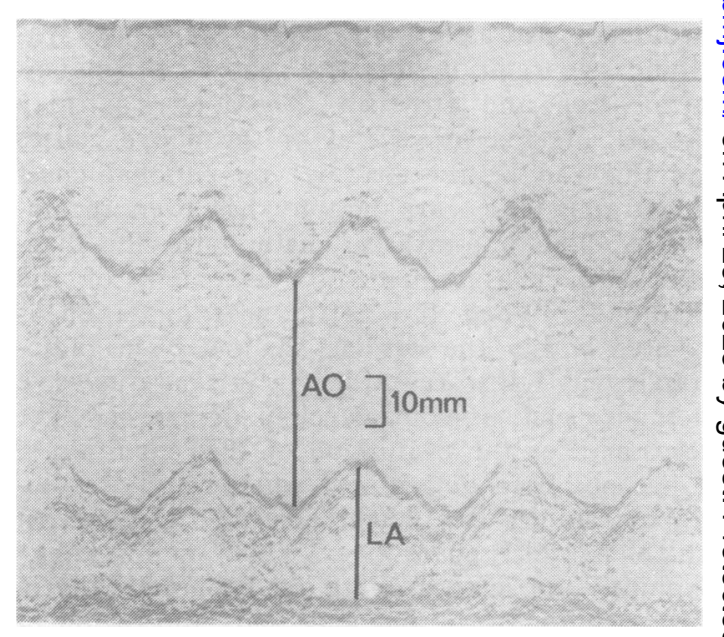

Fig 1 Echocardiogram of the aortic root $(A O)$ and left atrium (LA) just above the level of the aortic valve leaflets showing enlargement of the aortic root. 


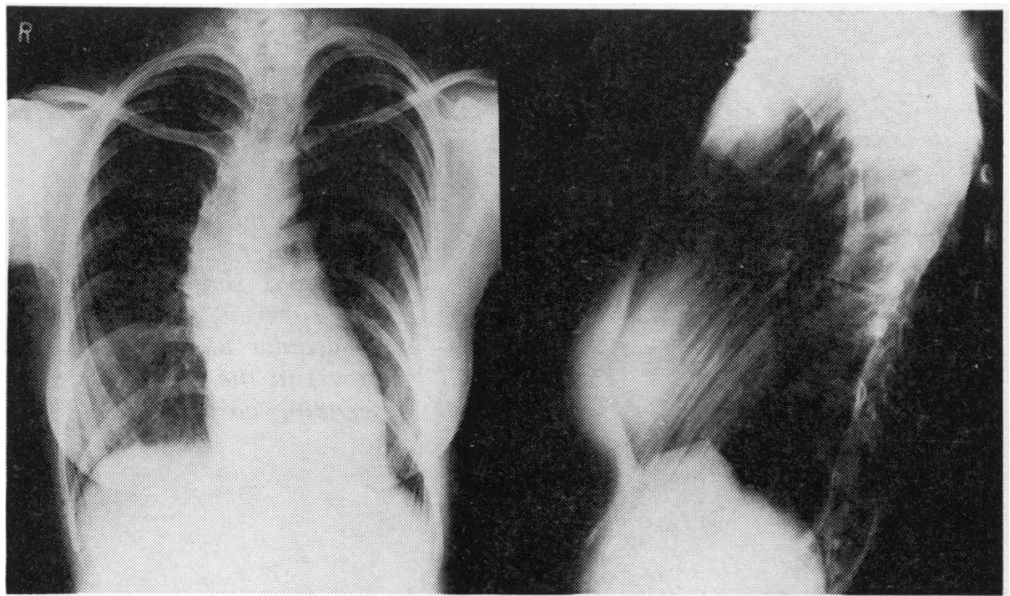

Fig 2 Postero-anterior (left) and left lateral (right) chest radiographs showing marked thoracic scoliosis but no evidence of aortic dilatation.

mediastinum and no evidence of aortic dilatation. Aortography confirmed mild aortic root dilatation involving the annulus of the aortic valve. There was no evidence of aortic regurgitation.

\section{PATIENT 2}

A 63-year-old man was referred from a neighbouring hospital complaining of mild precordial pain, wheeze, difficulty in swallowing, and hoarseness of the voice. He was known to have had syphilis at the age of 25 years. Echocardiographic studies showed a normal aortic root (fig 3) measuring $36 \mathrm{~mm}$ and normal aortic valve leaflets (fig 4). Conventional chest radiographs showed enlargement of the superior mediastinum in the postero-anterior view and rounded shadows in the ascending and descending aorta in the left lateral view (fig 5), compatible with saccular aneurysms. Aortography (fig 6) showed a large

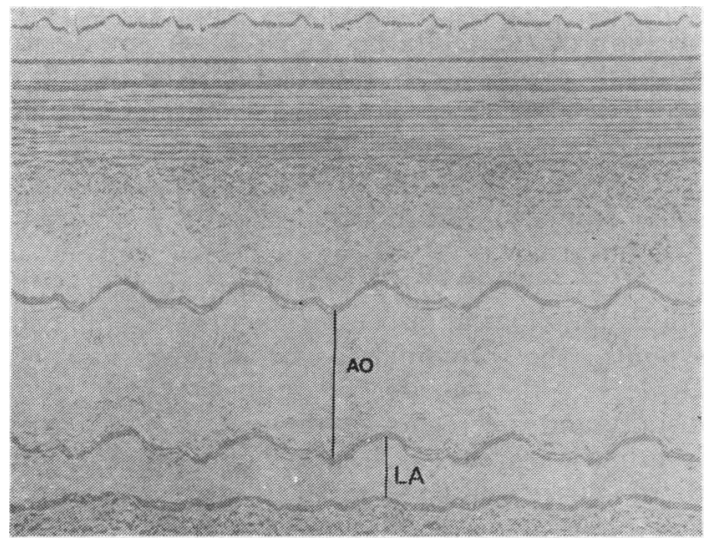

Fig 3 Echocardiogram in the patient with syphilis just above the aortic valve leaflets showing normal aortic root $(A O)$ and left atrium (LA). aneurysm giving the impression of a false aneurysm, originating from the ascending aorta, and fusiform dilatation and marked displacement of the ascending aorta to the left. The innominate artery was bowed around the upper medial surface of the aneurysm.

\section{Discussion}

Marfan's syndrome is inherited as an autosomal dominant trait and its association with aortic root dilatation is a sex-linked variable predominant in males $^{8}$ It has been speculated that aortic dilatation and mitral valve prolapse might be components of the forme fruste of Marfan's syndrome. ${ }^{89}$ It has been noted also ${ }^{3-5}$ that the region of the ascending aorta which contains the aortic annulus and sinuses of Valsalva lies within the pericardium and any dilatation at this level may

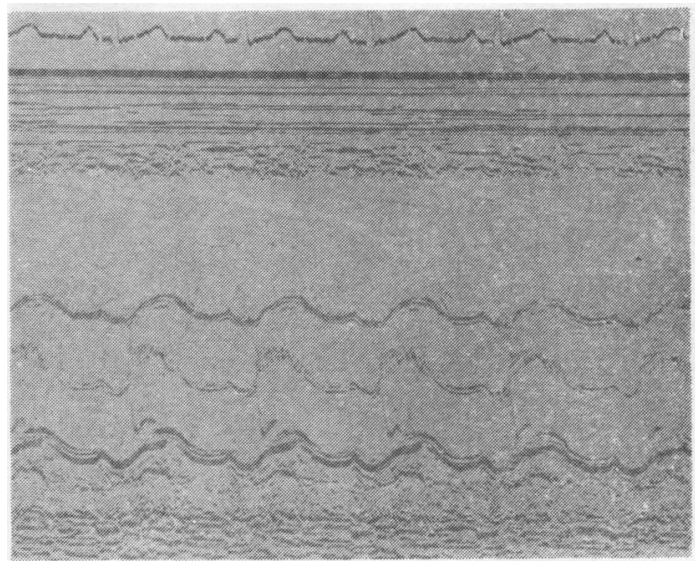

Fig 4 Echocardiogram in the patient with syphilis showing normal aortic valve leaflets. 


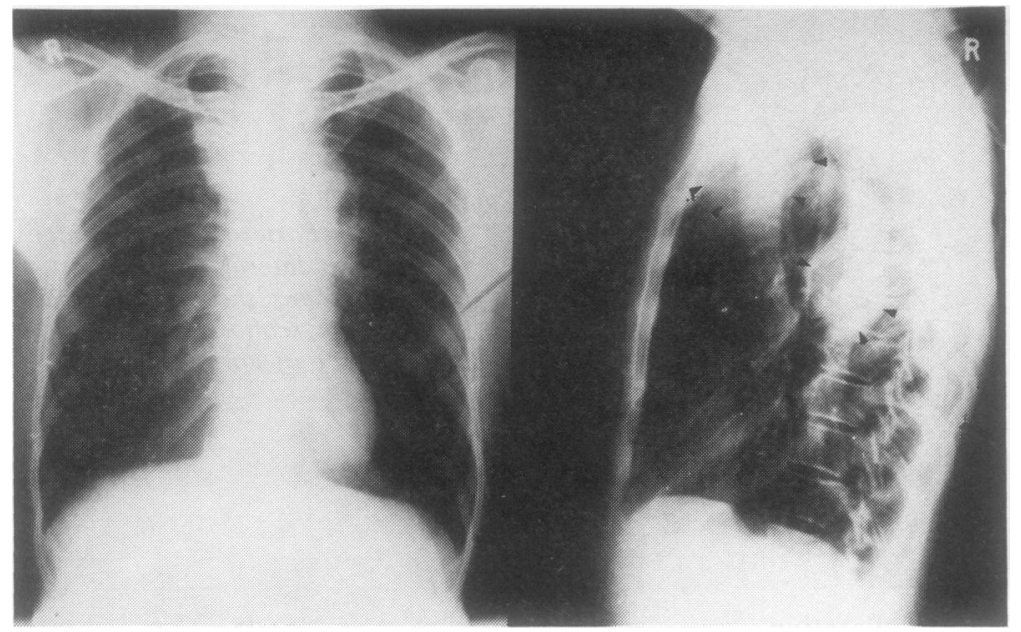

Fig 5 Postero-anterior (left) and left lateral (right) chest radiographs showing widening. of the superior mediastinum and saccular aneurysms (arrows) in the ascending and descending aorta.

not be apparent on the conventional chest radiographs. Echocardiography, however, visualises the cardiac structures and allows measurement of the aortic dimension at the level of the aortic leaflets, thereby identifying the proximal aortic

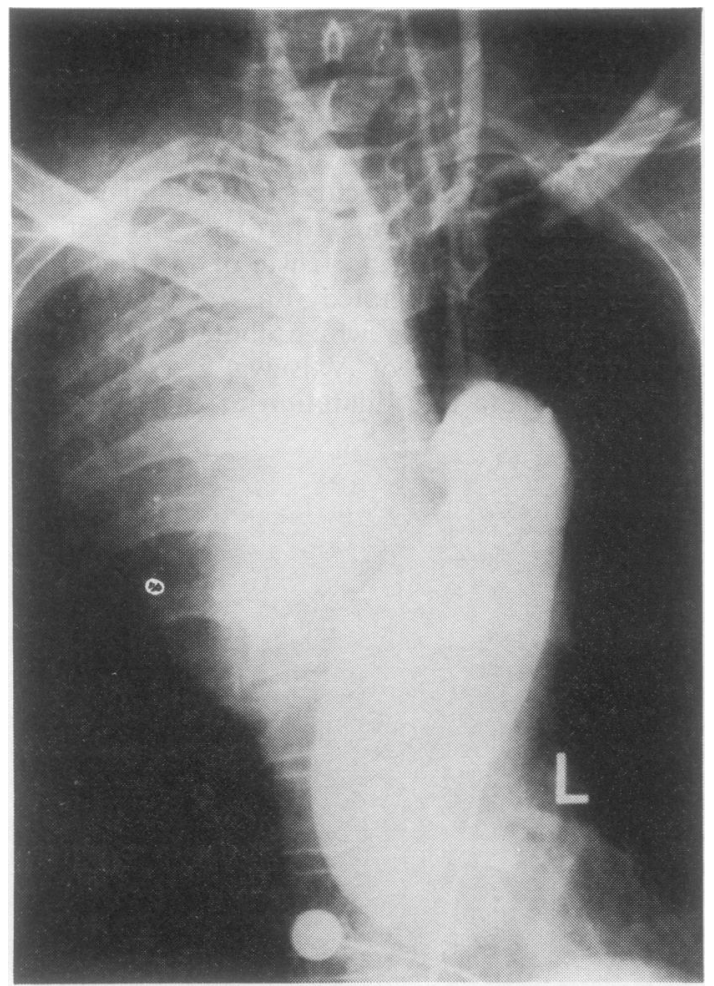

Fig 6 Aortogram showing fusiform dilation of the ascending aorta which is displaced to the left by a large aneurysm. root dilatation. ${ }^{6}$ When dilatation involves the ${ }^{\overparen{D}}$ distal portion of the ascending aorta it may be $\bar{\varnothing}$ evident on the chest radiographs. In the patient. with Marfan's syndrome, echocardiography revealed dilatation of the aortic root, while chest radiographs failed to do so. In this case the dilatation was minimal and did not cause aortic regurgitation. Proximal aortic dilatation in $\frac{\Phi}{\varnothing}$ Marfan's syndrome may progress with age and $\stackrel{2}{\vec{F}}$ lead to aortic regurgitation. ${ }^{10}$ Several authors 3 have noted that in Marfan's syndrome the aortic ring and the adjacent intrapericardial portion of the aorta are affected in any diffuse dilatation of the ascending aorta. ${ }^{11}$ Echocardiography, there-o fore, which easily demonstrates the aortic ring, may be preferable to chest radiography in the $\frac{0}{-}$ diagnosis and follow-up of patients with Marfan's 3 . stigmata. Since echocardiography is easily repeat-ô able, it is useful for studying the rate of pro- $₹$ gression of the dilatation and the efficacy of anyo medical or surgical treatment. Another fact $\square$ which makes the conventional radiograph lesso helpful in the diagnosis of aortic roat dilatation is that patients with Marfan's syndrome mayos suffer from scoliosis which obscures the picture. N

Echocardiography seems to be less reliable in ${ }_{\mathrm{C}}$ the diagnosis of aortic dilatation secondary tog other pathological processes such as syphilis. In syphilis, the proximal aorta is also affected. However, the aortic dilatation begins more? distally and the region of the sinuses of Valsalvato is usually spared. ${ }^{37}$ Chest radiography and aortography seem to be superior to echocardiography in syphilitic aortic dilatation because the dilata-这 tion lies outside the cardiac shadow. Distal aortic dilatation is difficult to detect by echocardiography because of the intervention of lung tissue. 


\section{References}

1 Gould SE. Pathology of the heart and the blood vessels. Third edition. Springfield, Illinois: Charles C Thomas, 1968; 980, 991.

2 Brown OR, Popp RL, Kloster FE. Echocardiographic criteria for aortic root dissection. Am J Cardiol 1975; 36:17-20.

3 Dinsmore RE, Jang GC. Roentgen diagnosis of aortic disease. Progr Cardiovasc Dis 1973; 16:151-85.

4 Steinberg I, Geller W. Aneurysmal dilatation of the aortic sinuses in arachnodactyly: diagnosis during life in three cases. Ann Intern Med 1955; 43:120-32.

5 Steinberg I. Dilatation of the aortic sinuses in the Marfan's syndrome: roentgen findings in five new cases. $A J R$ 1960; 83:302-19.

6 Come PC, Bulkley BH, McKusik VA, Fortuin NJ. Echocardiographic recognition of silent aortic root dilatation in Marfan's syndrome.
Chest 1977; 72:789-92.

7 Roberts WC, Dangel SC, Bulkley BH. Nonrheumatic valvular disease: a clinicopathologic study of 27 different conditions causing valvular dysfunction. Cardiovasc Clin 1973; 5:333-446.

8 Brown OR, DeMots H, Kloster FE, Roberts A, Menashe VD, Beals RK. Aortic root dilatation and mitral valve prolapse in Marfan's syndrome. An echocardiographic study. Circulation 1975; 52:651-7.

9 Rizzon O, Biasco G, Brindicci G, Mauro F. Familial syndrome of mid-systolic click and late systolic murmur. $B r$ Heart $J$ 1973; 35: 245-59.

10 McKusick VA. The cardiovascular aspects of Marfan's syndrome: a heritable disorder of the connective tissue. Circulation 1955; 11:321-42.

11 Lutman FC, Neel JV. Inheritance of arachnodactyly, ectopia lentis and other congenital anomalies (Marfan's syndrome) in the E family. Arch Ophthalmol 1949; 41:276-305. 